\title{
Inhibición de la formación de $\beta$-hematina de especies colombianas de Piper spp. y Calophyllum spp. como potenciales agentes antimaláricos
}

\author{
Ana M. Mesa ${ }^{1,2}$, Silvia Blair ${ }^{1} \&$ Carlos Peláez*2 \\ 1. Grupo de Investigación Malaria. Sede de Investigación Universitaria (SIU). Carrera 62 52-59. Torre 1. Lab. 610 \\ SIU. Facultad de Ciencias Exactas y Naturales, Instituto de Biología, Universidad de Antioquia. A.A 1226. Medellín, \\ Colombia; amaria.mesa@udea.edu.co, silviablairt@gmail.com \\ 2. Grupo Interdisciplinario de Estudios Moleculares GIEM. Facultad de Ciencias Exactas y Naturales, Instituto de \\ Química, Universidad de Antioquia. A.A 1226. Medellín, Colombia; carlos.pelaez@udea.edu.co \\ * Correspondencia
}

$$
\text { Recibido 03-V-2018. C Corregido 26-VII-2018. Aceptado 24-VIII-2018. }
$$

\begin{abstract}
Inhibition of $\beta$-hematin formation of Colombian species of Piper spp. and Calophyllum spp. as potential antimalarial agents. New antimalarial agents from plants are studied as alternatives in the treatment of malaria. The main antimalarials such as chloroquine have several mechanisms of action against parasites, one of which is the inhibition of polymerization of the heme group, a model that has allowed the design of new antimalarial candidates. In this sense the objective of this work was to evaluate extracts of genus Piper and Calophyllum plants on the inhibition capacity of $\beta$-hematin. Inhibitory concentrations of $\beta$-hematin are reported from 40 extracts of different polarity obtained from the species $P$. piedecuestanum, C. brasiliense, C. longinforium, and Calophyllum. sp. 19 extracts showed a greater potential to inhibit $\beta$-hematin with $\mathrm{IC}_{50}<3 \mathrm{mg} / \mathrm{ml}$. These activities mainly support future studies with the genus Calophyllum in the development and discovery of new antiplasmodial substances with known modes of action.
\end{abstract}

Key words: antiplasmodial; chloroquine; extracts; polymerization; heme group.

Mesa, A. M., Blair, S. \& Peláez, C. (2018). Inhibición de la formación de $\beta$-hematina de especies colombianas de Piper spp. y Calophyllum spp. como potenciales agentes antimaláricos. Revista de Biología Tropical, 66(4), 1412-1420.

La malaria es causada por un parásito de género Plasmodium, transmitido a los seres humanos por la picadura de la hembra del mosquito infectado de género Anopheles, que se reproduce en regiones que combinan calor, humedad y vegetación (OMS, 2017). Alrededor de 3200 millones de personas están en riesgo de contraer la enfermedad en 97 países y se estima que ocurrieron en el mundo en el 2015, 198 millones de casos (intervalo: entre 124 millones y 283 millones) (OMS, 2016). Las principales especies de Plasmodium que afectan al hombre son $P$. vivax y P. falciparum, la más peligrosa es $P$. falciparum, dada su patogenicidad y resistencia a los principales antimaláricos que en muchas ocasiones puede llevar a la muerte del individuo. Existen otras dos especies de importancia, $P$. malariae y $P$. ovale, que causan formas benignas de la malaria (Chin, 2005; Gelb, 2007). En sangre, se diferencian cuatro formas parasitarias asexuadas: anillos o trofozoítos jóvenes, trofozoítos maduros, esquizontes que al romperse liberan, merozoítos que son las formas que invaden los eritrocitos. (Londoño, Carmona, \& Blair, 2002; Olumese, 2006). La biología y bioquímica del Plasmodio son tópicos de interés científico en el diseño de nuevos fármacos antimaláricos (Liu et al., 2018). La mayoría de los medicamentos antimaláricos que están ahora en uso no son desarrollados sobre la base de blancos moleculares previamente identificados, sino 
productos del hallazgo de la actividad antimalárica de productos naturales utilizados tradicionalmente como tal (por ejemplo, quinina y la artemisinina). Recientemente, gracias a una mejor comprensión de la bioquímica de los parásitos de la malaria, se han podido identificar blancos potenciales para el diseño de nuevos medicamentos, los cuales han dado pautas sobre el modo de acción de muchos antimaláricos (Robert, Dechy-Cabaret, Cazelles, BenoitVical, \& Meunier, 2002; Fidock, Rosenthal, Croft, Brun, \& Nwaka, 2004).

Una de las vías y/o modos de acción de mayor relevancia en la literatura actual es la inhibición de biocristalización del grupo hemo generado principalmente por los derivados de la cloroquina (MacRaild, Pedersen, Anders, \& Norton, 2012). En la mayoría de su ciclo de vida en humanos, el parásito de género Plasmodium habita en los glóbulos rojos. Dentro de los eritrocitos, el evento inicial es la endocitosis de la hemoglobina, que es transportada hacia una organela denominada la vacuola digestiva ácida ( $\mathrm{pH}$ 5.2), donde los parásitos procesan entre un 60 y $80 \%$ de la hemoglobina presente en el glóbulo rojo. Aquí es donde la parte proteica (globina) es degradada por una serie de enzimas proteolíticas incluyendo plasmepsinas I, II, y IV, proteasa histoaspártica (HAP), falcipaínas 2 y 3 y falcilisinas para generar péptidos. Éstos son degradados a aminoácidos debido a la limitada capacidad del parásito para sintetizarlos, generando como subproducto el hemo libre ferriprotoporfirina (IX) (Fe (III) PPIX) o (FP); (el FP en la vacuola digestiva representa sólo el 3-5 \% del volumen total del parásito). Debido al medio ácido de la oxihemoglobina, el hierro se oxida de $\mathrm{Fe}^{2+}$ a $\mathrm{Fe}^{3+}$ con la consecuente producción de un 0.5 equivalente molar de $\mathrm{H}_{2} \mathrm{O}_{2}$ (Egan \& Ncokazi, 2005; Egan, 2008). Tanto el FP y $\mathrm{H}_{2} \mathrm{O}_{2}$ son moléculas tóxicas que el parásito necesita destruir o neutralizar. El FP "libre" en eritrocitos infectados con $P$. falciparum ha sido cuantificado en 0.1-0.4 mM. Incluso se ha llegado a estimar que la concentración local puede ser mucho mayor (Kumar, Guha, Choubey, Maity, \& Bandyopadhyay, 2007). Esto sugiere que el parásito vive en constante peligro, por lo que su mecanismo para la desintoxicación de FP debe ser preciso para evitar los efectos tóxicos de los residuos metabólicos de estos dos productos generados. Se estima que el parásito realiza una detoxificación mediante uniones coordinadas entre los grupos carboxilato y los $\mathrm{Fe}^{3+}$ presentes en la FP, propiciando un fenómeno de biocristalización entre estos grupos, generando así un producto de color carmelita oscuro denominado hemozoina o pigmento malárico, el cual no es tóxico debido a su insolubilidad en agua a pH fisiológico o ácido (Fitch, 2004). La inhibición de la desintoxicación FP, causa una acumulación de moléculas tóxicas de FP que eventualmente destruyen la integridad de las membranas del parásito de la malaria. $\mathrm{La}$ hemozoina se ha identificado químicamente mediante difracción de rayos $\mathrm{X}$ y espectroscopia mossbauer y la cual fue comparada con un compuesto de síntesis conocido como $\beta$-hematina. El entendimiento de este proceso de biocristalización de FP ha llevado a la identificación y búsqueda de una serie de posibles nuevos compuestos antimaláricos (Biamonte, Wanner, \& Le Roch, 2013).

El género Piper perteneciente a la familia Piperaceae, se distribuye en todo el mundo con aproximadamente 2300 especies. Este género tiene una gran importancia comercial y económica, para la industria de condimentos, farmacéutica, insecticida y se ha reportado una amplia gama de plantillas estructurales como lignanos, saponinas, fenoles flavonoides y alcaloides (Gupta, Arias, Correa, \& Lamba, 1979; Benevides, Sartorelli, \& Kato 1999; Prasad et al., 1995). Igualmente, Clusiaceae es una de las familias de plantas en la cual se han encontrado compuestos con actividad antimalárica, como xantonas y derivados del acilfloroglucinol. Se han reportado estos compuestos en plantas de los géneros Hypericum, Vismia y Garcinia. En la familia Clusiaceae, el género Calophyllum lo componen cerca de 200 especies, algunas de las cuales presentan reportes sobre compuestos de tipo cumarinas, xantonas y triterpenos con actividades antimalárica, analgésica, antiviral, antiulcerogénica, anticáncer, antibacteriana, y molusquicida (Noldin, Isaias \& Filho, 2006). Es así como los géneros Piper y Calophyllum 
pudieran ser fuentes de metabolitos secundarios, sus especies han sido poco estudiadas en el campo de la malaria y en los mecanismos de acción conocidos de los principales antimaláricos. Este trabajo tiene como objetivo evaluar la potencialidad de los extractos de diferente polaridad de las especies Piper piedecuestanum, C. brasiliense, C. longifolium y Calophyllum sp. en la inhibición de la formación de $\beta$-hematina.

\section{MATERIALES Y MÉTODOS}

Recolección del material vegetal: Se recolectaron tres tipos de muestras a partir del material vegetal: una muestra para espécimen de herbario, muestras como testigos de los especímenes recolectados y muestras de hojas y tallos para la obtención de los extractos. El espécimen para herbario fue procesado, depositado, caracterizado taxonómicamente en el herbario Universidad de Antioquia (HUA), y determinado como Piper piedecuestanum Trel. \& Yunck. (Voucher 164502) recolectado en Piedecuesta, Santander- Colombia. Calophyllum brasiliense Cambess recolectado en el departamento de Antioquia- Angelópolis (Voucher 162467), Calophyllum longifolium Willd recolectado en el departamento de Caldas- Norcasia (Voucher 166377) y una morfoespecie Calophyllum sp. recolectada en el departamento de Risaralda- Belén Umbría (Voucher 167392).

Preparación de los extractos de diferente polaridad: El material vegetal se sometió a un proceso de desecación a temperatura ambiente con aireación y sin exposición a la luz solar durante 10 días, posteriormente se pulverizó el material en un molino industrial hasta un tamaño de partícula de $5 \mathrm{~mm}$ para iniciar el proceso de extracción. Se llevó inicialmente el material vegetal molido a un proceso de percolación hasta agotamiento ( 5 días/3 veces), utilizando etanol (E) destilado como solvente con cada una de las plantas Piper piedecuestanum (PP) (0.236 kg mezcla de hojas y tallos), C. brasiliense Cambess (CB) $(0.015 \mathrm{~kg}$ de tallos), C. longifolium L (CI) $(0.016 \mathrm{~kg}$ mezcla de hojas y tallos). Por otra parte, el material vegetal seco y molido de $0.36 \mathrm{~kg}$ de hojas y tallos de P. piedecuestanum (PPHT); $1.64 \mathrm{~kg}$ de hojas de C. brasiliense Cambess (CBH); $1.74 \mathrm{~kg}$ de tallos de C. brasiliense Cambess (CBT); $0.13 \mathrm{~kg}$ de mezcla de hojas y tallos de C. brasiliense Cambess (CBHT); $0.27 \mathrm{~kg}$ hojas de $C$. longifolium $\mathrm{L}$. (CIH), $0,25 \mathrm{~kg}$ de tallos $C$. longifolium L. (CIT); $0.78 \mathrm{~kg}$ de mezcla hojas y tallos C. longifolium L. (CIHT); $0.51 \mathrm{~kg}$ de hojas Calophyllum sp. (CSH); $0.43 \mathrm{~kg}$ de tallos de Calophyllum sp. (CST); $0.75 \mathrm{~kg}$ de mezcla de hojas y tallos de Calophyllum sp. (CSHT), se extrajo sucesivamente por 10 días mediante percolación ( 3 x 4 L) hasta agotamiento con solventes de polaridad ascendente previamente destilados: éter de petróleo $(\mathrm{H})$, diclorometano (D), acetato de etilo (AE), y metanol (M). Los extractos fueron codificados con las iniciales de cada planta, tipo de material vegetal y solvente empleado. Después de tres días se comenzó la concentración del extracto a presión reducida en un rotavapor y posteriormente pesados para obtener los porcentajes de extracción según la ecuación 1.

$$
\% \text { de Extracción }=\frac{\text { Peso Extracto }}{\text { Peso Material Vegetal }} \times 100
$$

Inhibición de la biocristalización de 及-hematina: Para la evaluación de la inhibición de la biocristalización de $\beta$-hematina por los extractos se utilizó el método colorimétrico según Parapini et al. 2000, 2004; con algunas modificaciones (Parapini, Nicoletta, \& Erica, 2000, Parapini et al., 2004). Un total de 100 $\mu 1$ de una solución $1.562 \mathrm{mM}$ de cloruro de hemina disuelto en $\mathrm{NaOH} 0.2 \mathrm{M}$, se distribuyó en microplacas de 96 pozos $(0.4 \mu \mathrm{mol} /$ Pozo) con pipeta multicanal junto con $50 \mu 1$ de diferentes concentraciones de extractos en un rango: $5.0-0.078 \mathrm{mg} / \mathrm{ml}$ disueltos en dimetilsulfóxido (DMSO) al $100 \%$ y el control CQ se evaluó en un rango de 3.0-0.094 $\mathrm{mg} / \mathrm{ml}$. Se adicionó por triplicado cada concentración y se realizaron dos réplicas del ensayo. En los pozos control se adicionaron $50 \mu \mathrm{l}$ de DMSO para pozos sin tratamiento y control de cloroquina como referencia. La formación de $\beta$-hematina se inició mediante la adición de $50 \mu \mathrm{l}$ de ácido acético 2.175 M hasta un $\mathrm{pH}$ final de $2.8 \mathrm{y}$ 
$50 \mu 1$ de solución tampón de acetato de sodio $\left(\mathrm{C}_{2} \mathrm{H}_{3} \mathrm{NaO}_{2} \cdot 3 \mathrm{H}_{2} \mathrm{O}\right) 0.75 \mathrm{M}$. La placa se incubó a $37{ }^{\circ} \mathrm{C}$ durante $24 \mathrm{~h}$ y después se centrifugó a $4500 \mathrm{G}$ durante $1 \mathrm{~h}$. La fracción soluble del material fue descartada. El resto se resuspendió con $200 \mu \mathrm{l}$ de DMSO: $\mathrm{H}_{2} \mathrm{O}$ relación 1:1 para eliminar la hematina sin reaccionar. Las placas nuevamente se centrifugaron a $4500 \mathrm{G}$ durante 15 min por triplicado y el sobrenadante fue descartado. El precipitado, se disolvió en $\mathrm{NaOH}$ $0.2 \mathrm{M}$ para la cuantificación espectrofotométrica. Una alícuota de $50 \mu \mathrm{l}$ de cada pozo se transfirió a un nuevo plato con $150 \mu 1$ de $0.2 \mathrm{NaOH}$. Un blanco de muestra a las mismas concentraciones disuelto en $150 \mu \mathrm{l}$ de $0.2 \mathrm{NaOH}$ fue empleado. Antes de las lecturas se verifico la formación de $\beta$-hematina en un Spectronic 20 UV-Vis en el rango de $250-800 \mathrm{~nm}$. La cantidad de $\beta$-hematina inhibida por cada uno de los extractos se determinó midiendo la absorbancia (Abs) a $595 \mathrm{~nm}$ utilizando un lector de ELISA (BioRad) y se calculó el porcentaje de inhibición de $\beta$-hematina según la ecuación 2.

$\%$ de Extracción $=\frac{\text { Abs muestra }- \text { Abs Blanco de muestra }}{\text { Abs Control }} \times 100$

Análisis estadístico: Los resultados de los porcentajes de inhibición de los diferentes extractos y las concentraciones evaluadas son presentados como la media y su desviación estándar (Media \pm DS). Se realizó un modelo de regresión logística no lineal y un análisis de varianza de dos vías (ANOVA) con un nivel de confianza de $\mathrm{P}<0.05$ empleando el programa GraphPad Prism 5.

\section{RESULTADOS}

Los porcentajes de material obtenido de los extractos de las especies de Piper y Calophyllum con los diferentes solventes y las concentraciones inhibitorias medias sobre la producción de $\beta$-hematina se presentan en el cuadro 1 . Los mejores rendimientos de extracción lo tuvieron los extractos de diclorometano de la mezcla de tallos y hojas de las especies P. piedecuestanum (PPHTExtD) con $1.75 \%$ y para las especies de Calophyllum se presentaron máximos de rendimientos de extracción en porcentaje en peso con respecto al material vegetal en los extractos metanólicos de la especie C. brasiliense de $6.209 \%$ (CBHTExtM), de la especie $C$. longifolium fue $6.130 \%$ (CIHExtM) y de la especie Calophyllum sp. fue de $8.138 \%$ (CSHTExtM).

El ensayo colorimétrico de inhibición de $\beta$-hematina es simple, robusto y presenta un

CUADRO 1

Inhibición de $\beta$-hematina por los extractos de diferente polaridad de P. piedecuestanum y Calophyllum

TABLE 1

Inhibition of $\beta$-hematin by extracts of different polarities of P. piedecuestanum and Calophyllum

\begin{tabular}{|c|c|c|c|c|}
\hline \multirow{2}{*}{ Código } & \multirow{2}{*}{ Especie } & \multirow{2}{*}{ Extracto } & \multirow{2}{*}{$\begin{array}{l}\% \text { rendimiento de la extracción } \\
(\mathrm{g} \text { de extracto } / \mathrm{g} \text { de } \\
\text { material vegetal seco })^{* *}\end{array}$} & $\begin{array}{c}\mathrm{CI}_{50}(\mathrm{mg} / \mathrm{mL}) \\
\mathrm{X} \pm \mathrm{DE}^{* * *}\end{array}$ \\
\hline & & & & $\begin{array}{c}\text { Inhibición de } \\
\beta \text {-hematina**** }\end{array}$ \\
\hline PPHExtH & P. piedecuestanum & $\begin{array}{l}\text { Éter de petróleo, percolación hojas y tallos } \\
25^{\circ} \mathrm{C} \mathrm{ZP}^{*}: 5 \mathrm{~mm} 10 \text { días }\end{array}$ & 0.668 & $>5.00$ \\
\hline PPHTExtD & P. piedecuestanum & 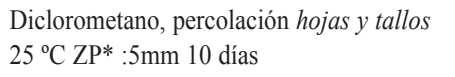 & 1.748 & $>5.00$ \\
\hline PPHTExtA & P. piedecuestanum & $\begin{array}{l}\text { Acetato Etilo, percolación hojas y tallos } 25 \\
{ }^{\circ} \mathrm{C} \text { ZP* }: 5 \mathrm{~mm} 10 \text { días }\end{array}$ & 1.116 & $>5.00$ \\
\hline PPHTExtM & P. piedecuestanum & $\begin{array}{l}\text { Metanol, percolación hojas y tallos } 25^{\circ} \mathrm{C} \\
\mathrm{ZP}^{*}: 5 \mathrm{~mm} 10 \text { días }\end{array}$ & 1.550 & $>5.00$ \\
\hline CBHTExtH & C. brasiliense & $\begin{array}{l}\text { Éter de petróleo, percolación hojas y tallos } \\
25^{\circ} \mathrm{C} Z \mathrm{Z}^{*}: 5 \mathrm{~mm} 10 \text { días }\end{array}$ & 1.791 & $2.610 \pm 0.032$ \\
\hline
\end{tabular}


CUADRO 1 (Continuación) / TABLE 1 (Continued)

\begin{tabular}{|c|c|c|c|c|}
\hline \multirow{2}{*}{ Código } & \multirow{2}{*}{ Especie } & \multirow{2}{*}{ Extracto } & \multirow{2}{*}{$\begin{array}{l}\% \text { rendimiento de la extracción } \\
\quad(\mathrm{g} \text { de extracto } / \mathrm{g} \text { de } \\
\text { material vegetal seco })^{* *}\end{array}$} & $\begin{array}{c}\mathrm{CI}_{50}(\mathrm{mg} / \mathrm{mL}) \\
\mathrm{X} \pm \mathrm{DE}^{* * *}\end{array}$ \\
\hline & & & & $\begin{array}{c}\text { Inhibición de } \\
\beta \text {-hematina**** }\end{array}$ \\
\hline CBHTExtD & C. brasiliense & $\begin{array}{l}\text { Diclorometano, percolación hojas y tallos } \\
25^{\circ} \mathrm{C} \mathrm{ZP} *: 5 \mathrm{~mm} 10 \text { días }\end{array}$ & 1.696 & $\mathrm{ND} * * * * *$ \\
\hline CBHTExtA & C. brasiliense & $\begin{array}{l}\text { Acetato Etilo, percolación hojas y tallos } \\
25^{\circ} \mathrm{C} \text { ZP*: } 5 \mathrm{~mm} 10 \text { días }\end{array}$ & 1.700 & $\mathrm{ND}$ \\
\hline CBHTExtM & C. brasiliense & $\begin{array}{l}\text { Metanol, percolación hojas y tallos } 25^{\circ} \mathrm{C} \\
\text { ZP*: } 5 \mathrm{~mm} 10 \text { días }\end{array}$ & 6.209 & $1.260 \pm 0.048$ \\
\hline CBTExtH & C. brasiliense & $\begin{array}{l}\text { Éter de petróleo, percolación tallos } 25^{\circ} \mathrm{C} \\
\mathrm{ZP} *: 5 \mathrm{~mm} 10 \text { días }\end{array}$ & 1.489 & $2.790 \pm 0.037$ \\
\hline CBTExtD & C. brasiliense & $\begin{array}{l}\text { Diclorometano, percolación tallos } 25^{\circ} \mathrm{C} \\
\mathrm{ZP} *: 5 \mathrm{~mm} 10 \text { días }\end{array}$ & 1.051 & $0.212 \pm 0.056$ \\
\hline CBTExtA & C. brasiliense & $\begin{array}{l}\text { Acetato Etilo, percolación tallos } 25^{\circ} \mathrm{C} \\
\mathrm{ZP} \text { : } 5 \mathrm{~mm} 10 \text { días }\end{array}$ & 1.685 & $2.660 \pm 0.028$ \\
\hline CBTExtM & C. brasiliense & $\begin{array}{l}\text { Metanol, percolación tallos } 25^{\circ} \mathrm{C} \\
\mathrm{ZP}^{*}: 5 \mathrm{~mm} 10 \text { días }\end{array}$ & 2.608 & $2.020 \pm 0.039$ \\
\hline CBHExtH & C. brasiliense & $\begin{array}{l}\text { Éter de petróleo, percolación hojas } 25^{\circ} \mathrm{C} \\
\mathrm{ZP} *: 5 \mathrm{~mm} 10 \text { días }\end{array}$ & 2.249 & ND \\
\hline CBHExtD & C. brasiliense & $\begin{array}{l}\text { Diclorometano, percolación hojas } 25^{\circ} \mathrm{C} \\
\mathrm{ZP} *: 5 \mathrm{~mm} 10 \text { días }\end{array}$ & 2.902 & ND \\
\hline CBHExtA & C. brasiliense & $\begin{array}{l}\text { Acetato Etilo, percolación hojas } 25^{\circ} \mathrm{C} \\
\mathrm{ZP}: 5 \mathrm{~mm} 10 \text { días }\end{array}$ & 2.241 & $0.266 \pm 0.046$ \\
\hline CBHExtM & C. brasiliense & $\begin{array}{l}\text { Metanol, percolación hojas } 25^{\circ} \mathrm{C} \\
\mathrm{ZP} *: 5 \mathrm{~mm} 10 \text { días }\end{array}$ & 3.719 & $>5.00$ \\
\hline CIHTExtH & C. longifolium & $\begin{array}{l}\text { Éter de petróleo, percolación hojas y } \\
\text { tallos } 25^{\circ} \mathrm{C} \mathrm{ZP}^{*}: 5 \mathrm{~mm} 10 \text { días }\end{array}$ & 1.529 & $0.908 \pm 0.068$ \\
\hline CIHTExtD & C. longifolium & $\begin{array}{l}\text { Diclorometano, percolación hojas y tallos } \\
25^{\circ} \mathrm{C} \mathrm{ZP*:} 5 \mathrm{~mm} 10 \text { días }\end{array}$ & 1.889 & $4.490 \pm 0.039$ \\
\hline CIHTExtA & C. longifolium & $\begin{array}{l}\text { Acetato Etilo, percolación hojas y tallos } 25 \\
{ }^{\circ} \mathrm{C} \text { ZP*: } 5 \mathrm{~mm} 10 \text { días }\end{array}$ & 1.661 & $0.429 \pm 0.028$ \\
\hline CIHTExtM & C. longifolium & $\begin{array}{l}\text { Metanol, percolación hojas y tallos } 25^{\circ} \mathrm{C} \\
\text { ZP*: } 5 \mathrm{~mm} 10 \text { días }\end{array}$ & 3.598 & $1.080 \pm 0.057$ \\
\hline CITExtH & C. longifolium & $\begin{array}{l}\text { Éter de petróleo, percolación tallos } 25^{\circ} \mathrm{C} \\
\mathrm{ZP}^{*}: 5 \mathrm{~mm} 10 \text { días }\end{array}$ & 0.500 & $0.982 \pm 0.079$ \\
\hline CITExtD & C. longifolium & $\begin{array}{l}\text { Diclorometano, percolación tallos } 25^{\circ} \mathrm{C} \\
\mathrm{ZP} * 5 \mathrm{~mm} 10 \text { días }\end{array}$ & 0.564 & $4.490 \pm 0.051$ \\
\hline CITExtA & C. longifolium & $\begin{array}{l}\text { Acetato Etilo, percolación tallos } 25^{\circ} \mathrm{C} \\
\mathrm{ZP} *: 5 \mathrm{~mm} 10 \text { días }\end{array}$ & 0.347 & ND \\
\hline CITExtM & C. longifolium & $\begin{array}{l}\text { Metanol, percolación tallos } 25^{\circ} \mathrm{C} \\
\mathrm{ZP}^{*}: 5 \mathrm{~mm} 10 \text { días }\end{array}$ & 0.931 & $1.080 \pm 0.057$ \\
\hline CIHExtH & C. longifolium & $\begin{array}{l}\text { Éter de petróleo, percolación hojas } 25^{\circ} \mathrm{C} \\
\mathrm{ZP} *: 5 \mathrm{~mm} 10 \text { días }\end{array}$ & 2.868 & $0.233 \pm 0.048$ \\
\hline CIHExtD & C. longifolium & $\begin{array}{l}\text { Diclorometano, percolación hojas } 25^{\circ} \mathrm{C} \\
\mathrm{ZP} *: 5 \mathrm{~mm} 10 \text { días }\end{array}$ & 2.026 & $0.299 \pm 0.056$ \\
\hline CIHExtA & C. longifolium & $\begin{array}{l}\text { Acetato Etilo, percolación hojas } 25^{\circ} \mathrm{C} \\
\mathrm{ZP}^{*}: 5 \mathrm{~mm} 10 \text { días }\end{array}$ & 1.298 & ND \\
\hline CIHExtM & C. longifolium & $\begin{array}{l}\text { Metanol, percolación hojas } 25^{\circ} \mathrm{C} \\
\mathrm{ZP}^{*}: 5 \mathrm{~mm} 10 \text { días }\end{array}$ & 6.130 & ND \\
\hline CSHTExtH & Calophyllum sp. & $\begin{array}{l}\text { Éter de petróleo, percolación hojas y tallos } \\
25^{\circ} \mathrm{C} \mathrm{ZP}^{*}: 5 \mathrm{~mm} 10 \text { días }\end{array}$ & 2.114 & $>5.00$ \\
\hline
\end{tabular}


CUADRO 1 (Continuación) / TABLE 1 (Continued)

\begin{tabular}{|c|c|c|c|c|}
\hline \multirow{2}{*}{ Código } & \multirow{2}{*}{ Especie } & \multirow{2}{*}{ Extracto } & \multirow{2}{*}{$\begin{array}{l}\% \text { rendimiento de la extracción } \\
\quad(\mathrm{g} \text { de extracto } / \mathrm{g} \text { de } \\
\text { material vegetal seco })^{* *}\end{array}$} & $\begin{array}{c}\mathrm{CI}_{50}(\mathrm{mg} / \mathrm{mL}) \\
\mathrm{X} \pm \mathrm{DE}^{* * *}\end{array}$ \\
\hline & & & & $\begin{array}{c}\text { Inhibición de } \\
\beta \text {-hematina**** }\end{array}$ \\
\hline CSHTExtD & Calophyllum sp. & $\begin{array}{l}\text { Diclorometano, percolación hojas y tallos } \\
25^{\circ} \mathrm{C} \mathrm{ZP} *: 5 \mathrm{~mm} 10 \text { días }\end{array}$ & 1.400 & $1.970 \pm 0.260$ \\
\hline CSHTExtA & Calophyllum sp. & $\begin{array}{l}\text { Acetato Etilo, percolación hojas y tallos } 25 \\
{ }^{\circ} \mathrm{C} \text { ZP*: } 5 \mathrm{~mm} 10 \text { días }\end{array}$ & 1.337 & ND \\
\hline CSHTExtM & Calophyllum sp. & $\begin{array}{l}\text { Metanol, percolación hojas y tallos } 25^{\circ} \mathrm{C} \\
\text { ZP*: } 5 \mathrm{~mm} 10 \text { días }\end{array}$ & 8.138 & $0.116 \pm 0.029$ \\
\hline CSTExtH & Calophyllum sp. & $\begin{array}{l}\text { éter de petróleo, percolación tallos } 25^{\circ} \mathrm{C} \\
\mathrm{ZP}^{*}: 5 \mathrm{~mm} 10 \text { días }\end{array}$ & 0.869 & $>5.00$ \\
\hline CSTExtD & Calophyllum sp. & $\begin{array}{l}\text { Diclorometano, percolación tallos } 25^{\circ} \mathrm{C} \\
\mathrm{ZP} *: 5 \mathrm{~mm} 10 \text { días }\end{array}$ & 0.526 & $1.970 \pm 0.089$ \\
\hline CSTExtA & Calophyllum sp. & $\begin{array}{l}\text { Acetato Etilo, percolación tallos } 25^{\circ} \mathrm{C} \\
\mathrm{ZP}^{*}: 5 \mathrm{~mm} 10 \text { días }\end{array}$ & 0.389 & ND \\
\hline CSTExtM & Calophyllum sp. & $\begin{array}{l}\text { Metanol, percolación tallos } 25^{\circ} \mathrm{C} \\
\mathrm{ZP} \text { : } 5 \mathrm{~mm} 10 \text { días }\end{array}$ & 1.145 & $0.115 \pm 0.029$ \\
\hline CSHExtH & Calophyllum sp. & $\begin{array}{l}\text { Éter de petróleo, percolación hojas } 25^{\circ} \mathrm{C} \\
\mathrm{ZP} *: 5 \mathrm{~mm} 10 \text { días }\end{array}$ & 3.271 & $0.436 \pm 0.037$ \\
\hline CSHExtD & Calophyllum sp. & $\begin{array}{l}\text { Diclorometano, percolación hojas } 25^{\circ} \mathrm{C} \\
\mathrm{ZP} \text { : } 5 \mathrm{~mm} 10 \text { días }\end{array}$ & 0.886 & ND \\
\hline CSHExtA & Calophyllum sp. & $\begin{array}{l}\text { Acetato Etilo, percolación hojas } 25^{\circ} \mathrm{C} \\
\text { ZP*: } 5 \mathrm{~mm} 10 \text { días }\end{array}$ & 1.113 & $0.442 \pm 0.023$ \\
\hline CSHExtM & Calophyllum sp. & $\begin{array}{l}\text { Metanol, percolación hojas } 25^{\circ} \mathrm{C} \\
\text { ZP*: } 5 \mathrm{~mm} 10 \text { días }\end{array}$ & 5.197 & $0.685 \pm 0.034$ \\
\hline
\end{tabular}

\footnotetext{
* ZP: Tamaño de partícula.

** Dato de una sola réplica.

*** X (Media) \pm DE (Desviación estándar).

$* * * *$ Control positivo Cloroquina sobre inhibición de $\beta$-hematina a $595 \mathrm{~nm} \mathrm{CI} \mathrm{m}_{50}=1.331 \pm 0.038 \mathrm{mg} / \mathrm{ml}$.

$* * * * * \mathrm{ND}=$ No determinado.
}

bajo costo-efectivo que puede ser empleado para un tamizaje de extractos a partir de plantas, con la finalidad de identificar sustancias promisorias como agentes antimaláricos (Vargas et al., 2011). La optimización de esta técnica permitió informar por primera vez la actividad inhibitoria de la $\beta$-hematina de 40 extractos obtenidos a partir de las especies P. piedecuestanum, C. brasiliense, C. longinforium, y Calophyllum sp. Para todos los extractos de las especies $P$. piedecuestanum, C. brasiliense, C. longifolium, Calophyllum sp. se encontró una adecuada relación concentración-respuesta con coeficientes de correlación cercanos a uno $\left(\mathrm{R}^{2}\right)$ estadísticamente significativos para todas las muestras evaluadas y con un nivel de confianza de P. En la figura 1 aparecen representados los extractos de diferente polaridad de las especies $P$. piedecuestanum y Calophyllum sp. que fueron capaces de inhibir la $\beta$-hematina con una $\mathrm{CI}_{50}<3 \mathrm{mg} /$ ml. 19 extractos fueron seleccionados por su potencial capacidad de inhibir la formación de $\beta$-hematina. Es de resaltar que los extractos con más potencial de inhibir la $\beta$-hematina fueron los extractos en diclorometano y acetato de etilo de las especies de Calophyllum. Nótese que los extractos; de tallos en diclorometano; de hojas en acetato de etilo C. de brasiliense; tallos y hojas en acetato de etilo; hojas en éter de petróleo; hojas en diclorometano de C. longinforium; hoja y tallo en metanol; hojas en acetato de etilo y en metanol de Calophyllum sp. fueron los que presentaron mayor actividad, 


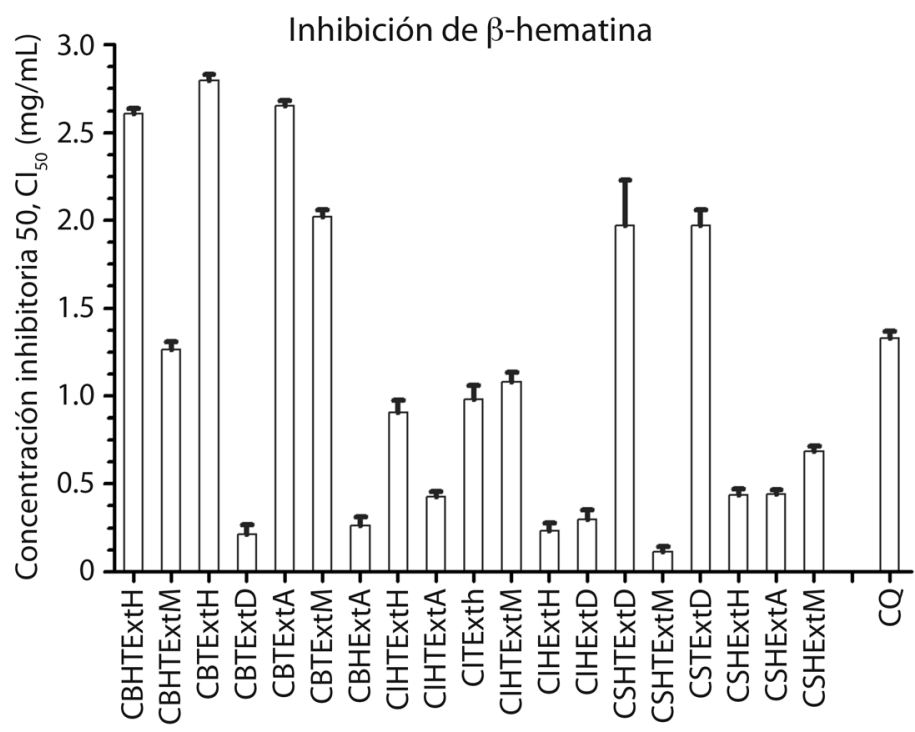

Tipos de extractos de diferente polaridad de Piper y Calophyllum

Fig. 1. Inhibición de la formación de la $\beta$-hematina por extractos de diferente polaridad de especies de género Piper y Calophyllum.

Fig. 1. Inhibition of formation of $\beta$-hematin by extracts of different polarity of species of genus Piper and Calophyllum.

indicando que los componentes presentes en estos extractos interaccionan fuertemente con la $\beta$-hematina con valores inferiores al del fármaco de referencia que fue la Cloroquina, $\mathrm{CQ}$ con una $\mathrm{CI}_{50}<1.33 \mathrm{mg} / \mathrm{ml}$.

\section{DISCUSIÓN}

Varios trabajos notifican la actividad inhibitoria por parte de extractos de plantas en la formación de la $\beta$-hematina. Osorio, Montoya, Muñoz, \& Arango (2006) presentaron el reporte de extractos de acetato de etilo de los tallos de Rollinia pittieri y Pseudomalmea boyacana (Annonaceae) con un $98 \%$ de inhibición de $\beta$-hematina. Igualmente, Osorio et al. (2005; 2006) reportaron 36 extractos de diferente polaridad de otras especies de la misma familia de Annonaceae (Annona muricata, Desmopsis panamensis, Rollinia exsucca, Rollinia pittieri) los cuales presentaron capacidad de inhibir la $\beta$-hematina con porcentajes de inhibición mayores al $85 \%$ cuando fueron evaluados a una concentración de $2.5 \mathrm{mg} / \mathrm{ml}$. Vargas et al., (2011) probaron un total de 40 moléculas y 219 extractos de plantas, encontrando buenas correlaciones con respecto a la especificidad (compuestos puros $85 \%$, extractos $93 \%$ ) y el valor predictivo (compuestos puros $72 \%$, extractos $50 \%$ ) que obtuvieron en comparación con la inhibición del crecimiento de Plasmodium en un modelo in vitro con una buena correlación. Por otra parte, se han reportado una serie de bisquinolinas, xantonas, una nueva clase de complejos metálicos de coordinación, 8-aminoquinolinas, medicamentos antimaláricos sintéticos como el azul de metileno, y derivados, que han demostrado tener actividades antimaláricas que están bien correlacionadas con sus capacidades para inhibir la polimerización del grupo hemo. Sin embargo, los detalles moleculares de esta inhibición son desconocidos (Asghari-Khiavi et al., 2011). La identificación de nuevas plantillas con mecanismos conocidos son necesarios para el desarrollo de tratamientos eficaces y seguros para la malaria, por lo que la evaluación de la 
inhibición de la formación de la $\beta$-hematina permite rastrear rutinariamente extractos de plantas con el objetivo final de identificar nuevas moléculas antimaláricas en una etapa inicial al descubrimiento de nuevos fármacos. Los positivos resultados obtenidos en este trabajo principalmente para los extractos de diclorometano y acetato de etilo de la especie C. brasiliense direccionan el estudio hacia la búsqueda de sustancias activas mediante la separación de sus principales componentes con el fin de encontrar sustancias con mecanismos similares a los antimaláricos convencionales.

En conclusión, este trabajo reporta la actividad inhibitoria de $\beta$-hematina de las especies P. piedecuestanum, Calophyllum brasiliense, Calophyllum longifolium y Calophyllum sp. Los extractos de la especie de $P$. piedecuestanum no presentaron capacidad de inhibición mientras que si se presentaron resultados promisorios en las especies de Calophyllum donde la especie más activa fue Calophyllum sp. con una $\mathrm{CI}_{50}=0.115 \mathrm{mg} / \mathrm{ml}$, seguido de C. brasiliense con una $\mathrm{CI}_{50}=0.212 \mathrm{mg} / \mathrm{ml} \mathrm{y}$ C. longifolium con una $\mathrm{CI}_{50}=0.233 \mathrm{mg} / \mathrm{ml}$, lo que permite continuar con los estudios de estas especies promisorias y explorar los metabolitos activos mediante un ensayo bioguiado.

\section{AGRADECIMIENTOS}

Los autores agradecen el apoyo financiero del Ministerio de agricultura a través del proyecto (No. 009-2007-V7552-38-07) y a Colciencias mediante las becas Francisco José de Caldas y al Grupo Malaria de la Universidad de Antioquia.

\section{RESUMEN}

Nuevos agentes antimaláricos a partir de plantas son estudiados como alternativas en el tratamiento de la malaria. Los principales antimaláricos como la cloroquina tienen varios mecanismos de acción contra parásitos, uno de ellos es la inhibición de polimerización del grupo hemo, modelo que ha permitido el diseño de nuevos candidatos antimaláricos. En este sentido, el objetivo de este trabajo fue evaluar extractos de plantas de género Piper y Calophyllum sobre la capacidad de inhibición de la $\beta$-hematina.
Se informa las concentraciones inhibitorias de la formación de $\beta$-hematina por parte de 40 extractos de diferente polaridad obtenidos a partir de las especies $P$. piedecuestanum, C. brasiliense, C. longinforium, y Calophyllum. sp. 19 extractos mostraron un mayor potencial para inhibir la formación de $\beta$-hematina con $\mathrm{CI}_{50}<3 \mathrm{mg} / \mathrm{ml}$. Estas actividades respaldan principalmente, futuros estudios con el género Calophyllum, en el desarrollo y descubrimiento de nuevas sustancias antiplasmodiales con modos de acción conocido.

Palabras clave: antiplasmodial; cloroquina; extractos; polimerización; grupo hemo.

\section{REFERENCIAS}

Asghari-Khiavi, M., Vongsvivut, J., Perepichk, I., Mechler, A., Wood, B. R., McNaughton, D., \& Bohle, D. S. (2011). Interaction of quinoline antimalarial drugs with ferriprotoporphyrin IX, a solid state spectroscopy study. Journal of Inorganic Biochemistry, 105(12), 1662-1669.

Benevides, P. J., Sartorelli, P., \& Kato, M. J. (1999). Phenylpropanoids and neolignans from Piper regnellii. Phytochemistry, 52(2), 339-343.

Chin, J. (2001). El control de las enfermedades transmisibles ( $17^{\circ}$ Edición). Maryland, EUA: Organización Panamericana de la Salud.

Christopher, A., MacRaild, M., Pedersen, R., \& Anders, R. (2012). Lipid interactions of the malaria antigen merozoite surface protein 2. Biochimica et Biophysica Acta, 1818, 2572-2578.

Egan, T. J., \& Ncokazi, K. K. (2005). Quinoline antimalarials decrease the rate of $\beta$-hematin formation. Journal of Inorganic Biochemistry, 99(7), 1532-1539.

Egan, T. J. (2008). Recent advances in understanding the mechanism of hemozoin (malaria pigment) formation. Journal of Inorganic Biochemistry, 102(5-6), 1288-1299.

Fidock, D. A., Rosenthal, P. J., Croft, S. L., Brun, R., \& Nwaka, S. (2004). Antimalarial drug discovery: efficacy models for compound screening. Nature Reviews Drug Discovery, 3(6), 509-520.

Fitch, C. D. (2004). Ferriprotoporphyrin IX, phospholipids, and the antimalarial actions of quinoline drugs. Life Sciences, 74(16), 1957-1972.

Gelb, M. H. (2007). Drug discovery for malaria: a very challenging and timely endeavor. Current Opinion in Chemical Biology, 11, 440-445.

Gupta, M. P., Arias, T. D., Correa, M., \& Lamba, S. S. (1979). Ethnopharmacognositc observations on 
Panamanian medicinal plants. Part I. Quarterly Journal of Crude Drug Research, 17(3-4), 115-130.

Kumar, S., Guha, M., Choubey, V., Maity, P., \& Bandyopadhyay, U. (2007). Antimalarial drugs inhibiting hemozoin ( $\beta$-hematin) formation: A mechanistic update. Life Sciences, 80(9), 813-828.

Liu, X., Wang, Y., Liang, J., Wang, L., Qin, N., Zhao, Y., \& Zhao, G. (2018). In-depth comparative analysis of malaria parasite genomes reveals protein-coding genes linked to human disease in Plasmodium falciparum genome. BMC Genomics, 19(1), 312.

Londoño, B., Carmona, J., \& Blair, S. (2002). Comparación de los métodos Optimal y gota gruesa para el diagnóstico de malaria en una zona endémica sin epidemia. Biomédica, 22(4), 466-475.

MacRaild, C. A., Pedersen, M. Ø., Anders, R. F., \& Norton, R. S. (2012). Lipid interactions of the malaria antigen merozoite surface protein 2. Biochimica et Biophysica Acta (BBA)-Biomembranes, 1818(11), 2572-2578.

Noldin, V. F., Isaias, D. B., \& Filho, C. V. (2006). Calophyllum Genus: Chemical and pharmacological importance. Química Nova, 29(3), 549-554.

Olumese, P. (2005). Epidemiology and surveillance: changing the global picture of malaria-myth or reality? Acta Tropica, 95(3), 265-269.

Organización Mundial de la Salud (OMS). (2016). Estrategia Técnica Mundial contra la Malaria 20162030. Organización Mundial de la Salud. Ginevra, Suiza: Recuperado de http://www.who.int/malaria/ publications/atoz/9789241564991/es/

Organización Mundial de la Salud (OMS). (2017). World malaria report 2017. Geneva, Suiza: World Health Organization Press. Retrieved from http://www.who. int/malaria/publications/world-malaria-report-2017/ report/en/

Osorio, E. J., Arango, G., García, E., Muñoz, K., Ruiz, G., Gutiérrez, D., \& Paco, M. A. (2005). Actividad
Antiplasmódica In Vitro e Inhibición de la Formación de la $\beta$-Hematina de Plantas Colombianas de la Familia Annonaceae. Acta Farmacéutica Bonaerense, 24(4), 527-32.

Osorio, E., Arango, G., García, E., Muñoz, K., Ruiz, G., Gutiérrez, D., \& Giménez, A. (2005). Actividad antiplasmódica in vitro e inhibición de la formación de la $\beta$-hematina de plantas colombianas de la familia Annonaceae. Acta Farmacéutica Bonaerense, 24(4), 527-532.

Osorio, E. J., Montoya, G., Muñoz, K., \& Arango, G. (2006). Actividad antiplasmodial de alcaloides aporfínicos de Rollinia pittieri y Pseudomalmea boyacana (Annonaceae). Vitae, 13(1), 49-54.

Parapini, S., Basilico, N., Mondani, M., Olliaro, P., Taramelli, D., \& Monti, D. (2004). Evidence that haem iron in the malaria parasite is not needed for the antimalarial effects of artemisinin. FEBS letters, 575(1-3), 91-94.

Parapini, S., Basilico, N., Pasini, E., Egan, T. J., Olliaro, P., Taramelli, D., \& Monti, D. (2000). Standardization of the physicochemical parameters to assess in vitro the $\beta$-hematin inhibitory activity of antimalarial drugs. Experimental parasitology, 96(4), 249-256.

Prasad, A. K., Tyagi, O. D., Wengel, J., Boll, P. M., Olsen, C. E., Bisht, K. S., \& Parmar, V. S. (1995). Neolignans and a lignan from Piper clarkii. Phytochemistry, 39(3), 655-658.

Robert, A., Dechy-Cabaret, O., Cazelles, J., Benoit-Vical, F., \& Meunier, B. (2002). Recent advances in malaria chemotherapy. Journal of the Chinese Chemical Society, 49(3), 301-310.

Vargas, S., Ioset, K. N., Hay, A. E., Ioset, J. R., Wittlin, S., \& Hostettmann, K. (2011). Screening medicinal plants for the detection of novel antimalarial products applying the inhibition of $\beta$-hematin formation. Journal of Pharmaceutical and Biomedical Analysis, 56(5), 880-886. 